\title{
Spray- and Spin-Assisted Layer-by-Layer Assembly of Copper Nanoparticles on Thin-Film Composite Reverse Osmosis Membrane for Biofouling Mitigation
}

Wen $\mathrm{Ma}^{1}$, Adel Soroush ${ }^{1}$, Tran Van Anh Luong ${ }^{1}$, Gregory Brennan ${ }^{1}$, Md. Saifur Rahaman ${ }^{1 *}$, Bahareh Asadishad ${ }^{2}$, Nathalie Tufenkji ${ }^{2}$

\author{
Water Research \\ Revised: April 11, 2016 \\ ${ }^{I}$ Department of Building, Civil and Environmental Engineering \\ Concordia University, Montreal, QC, H3G 1 M8 \\ ${ }^{2}$ Department of Chemical Engineering, McGill University, \\ Montreal, QC, Canada H3A OC5
}

* Corresponding author: Md. Saifur Rahaman, Email: saifur.rahaman@concordia.ca, Phone: 5148482424 Ext 5058. 
Abstract: Copper nanoparticles (CuNPs) have long been considered as highly effective biocides; however, the lack of suitable methods for loading CuNPs onto polymeric membranes is recognized as being one of the primary reasons for the limited research concerning their application in membrane industries. A highly efficient spray- and spin-assisted layer-by-layer (SSLbL) method was developed to functionalize the TFC polyamide RO membranes with controllable loading of CuNPs for biofouling control. The SSLbL method was able to produce a uniform bilayer of polyethyleneimine-coated CuNPs and poly(acrylic) acid in less than one minute, which is far more efficient than the traditional dipping approach $(25-60 \mathrm{~min})$. The successful loading of CuNPs onto the membrane surface was confirmed by XPS analysis. Increasing the number of bilayers from 2 to 10 led to an increased quantity of CuNPs on the membrane surface, from 1.75 to $23.7 \mu \mathrm{g} \mathrm{cm}^{-2}$. Multi-layer coating exhibited minor impact on the membrane water permeation flux (13.3\% reduction) while retaining the original salt rejection ability. Both static bacterial inactivation and cross-flow filtration tests demonstrated that CuNPs could significantly improve anti-biofouling property of a polyamide membrane and effectively inhibit the permeate flux reduction caused by bacterial deposition on the membrane surface. Once depleted, CuNPs can also be potentially regenerated on the membrane surface via the same SSLbL method.

Keywords: Reverse osmosis, biofouling, copper nanoparticles, spray-and spin-assisted layer-by-layer (SSLbL) self-assembly 


\section{Introduction}

The global fresh water crisis is universally considered to be one of the most critical challenges currently faced by the international community (Rahaman et al. 2012). Water reuse and desalination have been considered as the most feasible ways to alleviate the issue. Through superior water purification performances, the occupation of a smaller physical footprint, and boasting lower construction costs (Greenlee et al. 2009), reverse osmosis (RO) has proven to be a competitive unit for the various types of waste water treatment and the brackish/seawater desalination. The number of desalination plants based on the RO process continues to increase together with a decrease in the energy consumption (Elimelech and Phillip 2011) makes this technology ever more appealing. Therefore, it should be noted that RO should play an important role in the future fresh water supply.

Since their development in the 1970s, thin film composites (TFC) have been treated as the core structure of RO membranes. The benchmark material (Elimelech and Phillip 2011), polyamide (PA), is used as the top active layer of a TFC membrane with a thickness of only 50-230 nm, which contributes to its exceptional performance with respect to water permeation and salt rejection (Kang and Cao 2012). However, the major drawback of the polyamide membrane is biofouling, which limits its widespread application (Rana and Matsuura 2011). Biological substances are ubiquitous in any water treatment environment, and once they adhere onto the membrane, bacteria and metabolites generated during cell growth will lead to biofilm formation on the membrane surface, causing irreversible degradation of membrane material. Biofouling not only affects the membrane's lifespan but also adds an energy burden and consequently becomes a severe obstacle constraining current RO desalination techniques (Mi and Elimelech 2010). 
Antibacterial polymers (Coneski et al. 2013, Ma et al. 2015) are commonly used as coating materials to improve membrane fouling resistance; however, their anti-bacterial activity decreases over time because of a deposited fouling layer duringthe "contact killing" process (Ben-Sasson et al. 2014a). With growing interest in nanomaterials, carbon nanotubes (CNT) (Tiraferri et al. 2011, Vecitis et al. 2011) and graphene oxide (GO) (He et al. 2015) have become widely studied membrane antifouling agents; while, similar to the biocidal polymers, CNT/GO inactive cells via "contact killing", which would gradually compromise the biocidal function within a short time. Unlike biocidal polymers, metal nanoparticles (NPs) and their oxides/ionic forms, e.g., silver and copper (Das et al. 2011), can not only prevent the growth of cell colonies on the contacting surface but also release bacteria-inactivating ions into the feed solution, thus inhibiting bacterial reproduction (Mansouri et al. 2010). These advantages have generated significant interest in membrane fouling control research. In comparison to other metal NPs, silver possesses better stability properties and is less prone to oxidation, thus, it is one of the most extensively explored option for membrane bio-fouling mitigation (Soroush et al. 2015, Soroush et al. 2016). However, with a relatively high cost, the financial impact of employing silver significantly limits its widespread application (Ben-Sasson et al. 2016).

Copper is another popular biocide that costs significantly less than silver. Copper nanoparticles (Ben-Sasson et al. 2014a), copper oxide nanoparticles (Ahamed et al. 2014) and copper ions (Dwidjosiswojo et al. 2011) have all demonstrated antimicrobial properties against various bacterial strains; while, only a handful of studies have focused on using copper as a coating material to improve fouling resistance in membrane processes. Ben-Sasson et al. (Ben-Sasson et al. 2014a) incorporated polyethyleneimine (PEI)-coated copper nanoparticles (CuNPs) onto an RO membrane surface through electrostatic interactions; a significant reduction (80-96\%) of live 
bacteria attached onto the membrane demonstrated the potential of using copper to replace silver for membrane biofouling control. However, despite the aforementioned benefits, the modification method used (i.e., dip-coating) is considerably time consuming (Morton et al. 2013). Furthermore, the number of CuNPs loaded onto the membrane surface via the dip-coating method is uncontrollable, and the membrane is likely to lose its antimicrobial functionality through the gradual dissolution of the mono-layered CuNPs. Thus, the development of an efficient method to controllably load the biocide and thereby inhibit biofouling for more sustainable applications is needed.

Blending metal NPs into the membrane casting solution (Liu et al. 2013), anchoring NPs into the membrane structure via the cold spray technique (Dumée et al. 2015), and developing a NP coating onto the membrane surface through plasma treatment (Mauter et al. 2011) all appreciably improve the antibacterial activities for some ultrafiltration (UF) and microfiltration (MF) membranes. However, these NP loading methods are not suitable for the surface modification of a RO membrane, since they result in irretrievable damage of the polyamide active layer. Therefore, the salt rejection of the membrane would decrease with the gradual release/consumption of the metal particles and the regeneration of the NPs would also be challenging. Layer-by-layer (LbL) selfassembly is an effective strategy for fabricating functionalized multilayers on a membrane surface (Rajesh et al. 2014), and the number of functional units can be precisely controlled by manupulating the number of multi-layers (Tiraferri et al. 2011). Since no adverse chemical reactions take place during the procedure, the properties of the original membrane are not altered by this multiple film loading modification (Chen et al. 2013). Although some researchers have implemented LbL assembly to apply nanoparticles for biofouling control, the manual dipping LbL assembly operation is time consuming, and the coating proves not as uniform as would be expected 
(Morton et al. 2013). These unsatisfactory results present an opportunity to improve the coating efficiency and quality of the LbL modification method. Automatic spray- and spin-assisted LbL (SSLbL) processes have been reported to produce uniform nanotube/nanowire electrodes (Gittleson et al. 2015) and polymer coating on glass plates (Gittleson et al. 2012); however, to the best or our knowledge, it has never been used for membrane surface modification.

In this study, the efficient SSLbL method was applied to assemble CuNP functionalized antibacterial coatings on a commercial RO membrane in a controllable manner. This antifouling coating consists of multi-layers that employ PEI-coated CuNPs as a polycation and PAA as a polyanion. By taking advantage of the negative charge on the polyamide surface, the multi-films are firmly deposited onto the membrane and held in place by the resulting electrostatic interactions. The successful modification of a commercial RO membrane was examined, and the modified membrane surface properties were assessed. The effect of the modification on membrane performance was evaluated through water permeability and salt rejection experiments. Moreover, the biocidal properties of the modified membrane were evaluated through static bacterial cell inactivation and cross-flow cell filtration tests. The SSLbL methodology provides a uniform coating of CuNPs on the membrane surface, offers controllable particle loading and also presents a high modification efficiency (32 sec per bilayer deposition) compared with manual dip-coating LbL modification (25-60 min per bilayer deposition) (Chen et al. 2013), indicating the potential for its practical application in commercial anti-biofouling membrane modification practices. 


\section{Materials and Methods}

\subsection{Chemicals and materials}

Copper sulfate $\left(\mathrm{CuSO}_{4}\right)$, sodium borohydride $\left(\mathrm{NaBH}_{4}\right)$, nitric acid $\left(\mathrm{HNO}_{3}\right)$, polyethylenimine $(\mathrm{PEI})$ (branched, MW=25 kDa), isopropyl alcohol, hydrochloric acid ( $\mathrm{HCl}$ ) and poly(acrylic acid) (PAA) were all purchased from Sigma-Aldrich (Oakville, ON, Canada), and all solutions were prepared in deionized (DI) water. The UTC-82C seawater desalination membrane was obtained from Toray Company (Poway, CA, USA).

\subsection{Preparation of the PEI-coated copper nanoparticles (CuNPs)}

Positively charged PEI-CuNPs were synthesized via the wet chemical reduction method (Figure 1.A) following a published protocol (Ben-Sasson et al. 2014a). Briefly, $10 \mathrm{~mL}$ of $50 \mathrm{mM} \mathrm{CuSO}_{4}$ was added to $30 \mathrm{~mL}$ of $0.066 \mathrm{mM}$ PEI solution. After a $5 \mathrm{~min}$ reaction with magnetic stirring, 10 $\mathrm{mL}$ of $100 \mathrm{mM} \mathrm{NaBH} 4$ was gradually added into the solution, reacting for approximately $25 \mathrm{~min}$. The formed CuNPs were subsequently dialyzed for $20 \mathrm{~h}$ to remove unreacted ions.

\subsection{Loading CuNPs on the membrane surface via the layer-by-layer (LbL) self-assembly}

The RO membrane was stored as received in DI water at $4{ }^{\circ} \mathrm{C}$. To modify the active layer, the membrane coupon was immersed in $20 \%$ isopropanol solution for $20 \mathrm{~min}$. Then, the pretreated membranes were rinsed several times and soaked into in DI water until use. The Cee® 200XD Model spray/puddle developer (Brewer Science Inc. Rolla, MO, USA) with spray- and spinassisted coating functionalities was used to achieve uniform layer-by-layer modification. A picture of the coating system is presented in Figure S1, and the schemes of the spray- and spin-assisted PEI-CuNPs/PAA layer-by-layer coating modification processes are displayed in Figure 1.B-C. 
Briefly, a $10 \mathrm{~cm} \times 10 \mathrm{~cm}$ membrane coupon was adhered onto a polycarbonate plate and spun at $2000 \mathrm{rpm}$ while being spray-coated at $2.1 \mathrm{bar}$ (30 psi), alternating between the positively charged PEI-CuNPs (pH 8.3) and negatively charged PAA solution $(\mathrm{pH} 3,1 \mathrm{~g} / \mathrm{L})$ in increments of $3 \mathrm{~s}$ at 5 $\mathrm{mL} / \mathrm{s}$. Between each layer deposition, the membrane was rinsed with DI water for $3 \mathrm{~s}$ at $5 \mathrm{~mL} / \mathrm{s}$ and then air-dried for $10 \mathrm{~s}$ (with only spinning). This process completed one cycle of LbL deposition to form a single bilayer of PEI-CuNPs/PAA. The same process was then repeated until a desired number of bilayers was achieved.

\section{FIGURE 1.}

\subsection{Membrane characterization}

The thickness of the bilayer coating was evaluated by forming exactly the same number of LbL multi-films on the pristine silicon wafer and then analyzed by a profilometer (Dektak XT, Bruker, Germany). X-ray photoelectron spectroscopy (XPS) (ThermoFisher Scientific K-Alpha, Waltham, MA, USA) analysis of the membrane was performed using a monochromatized Al-KaX-ray source $\mathrm{v}=1350 \mathrm{eV}$ with a spot size of $400 \mu \mathrm{m}$. The surface zeta potential of the membrane was assessed by an electrokinetic analyzer (EKA) (Anton Paar, Graz, Austria) using $1 \mathrm{mM} \mathrm{KCl}$ solution, and the $\mathrm{pH}$ was adjusted from 4 to 10 using $1 \mathrm{M} \mathrm{NaOH}$ and $1 \mathrm{M} \mathrm{HCl}$. The membrane surface roughness was evaluated using an atomic force microscope (AFM) (NanoINK Inc. Skokie, IL, USA) in tapping mode. The average roughness values were calculated by analyzing the results of three randomly selected scanned positions on the membrane surface $(10 \mu \mathrm{m} \times 10 \mu \mathrm{m})$ by using the software 'Gwyddion'. The water contact angle of the membrane was measured by a Video Contact Angle system (VCA, AST Products, Inc., Billerica, MA, USA). At least three position were selected on each membrane surface to obtain the average contact angle value. 
To quantify the CuNPs loaded onto the membrane surface, a sample with an area of $3.8 \mathrm{~cm}^{2}$ was cut from a modified membrane and then immersed into $10 \mathrm{~mL}$ solution containing $1 \% \mathrm{HNO}_{3}$ and $0.5 \% \mathrm{HCl}$. The total amount of CuNPs released in the acidic solution were quantified by atomic absorption spectroscopy (SpectraAA 220 FS, Agilent Technologies, Inc. Santa Clara, CA, USA).

\subsection{Evaluation of membrane perm-selectivity}

A standard laboratory-scale RO cross flow filtration system (Figure S2) was used to test the water permeate flux and salt rejection. Specifically, a membrane with an effective area of $20.02 \mathrm{~cm}^{2}$ was compacted overnight at 27.6 bar (400 psi) until a steady water permeate flux was reached. The water permeate flux was monitored with a digital flow meter (Liquid Flow Meter SLI-2000, Sensirion Inc.CA,USA), and the salt rejection was assessed by measuring the rejection of $50 \mathrm{mM}$ $\mathrm{NaCl}$ solution using a calibrated conductivity meter (Oakton Instruments, Vernon Hills, IL, USA). All filtration experiments were performed at $20.0 \pm 0.5{ }^{\circ} \mathrm{C}$ with a cross-flow velocity of $21.4 \mathrm{~cm} / \mathrm{s}$.

\subsection{Observation of membrane antimicrobial property}

Static bacterial inactivation tests using three different bacterial strains were performed to evaluate the antimicrobial properties of the membrane. Enterococcus faecalis (ATCC 29212) was used as a representative Gram positive organism, and Escherichia coli D21f2 and Escherichia coli O157:H7 (ATCC 700927) were used as representative nonpathogenic and pathogenic Gram negative strains, respectively. The static cell inactivation test was performed according to the following protocol. First, a single colony of each bacterial strain was added to $20 \mathrm{~mL}$ sterile lysogeny broth (LB) solution, and incubated overnight with shaking (70 rpm) at $35^{\circ} \mathrm{C}$. Next, the bacterial suspension was poured in a sterile centrifuge tube and centrifuged at $5000 \mathrm{~g}$ (Mandel Multifuge X3R, 75003603) for 10 min in 2 sequential cycles. After each centrifugation cycle, the 
supernatant was discarded, and the remaining bacterial cell pellet was resuspended by adding 20 $\mathrm{mL}$ of $0.9 \% \mathrm{NaCl}$ and vortexing. Finally, the bacterial suspension was diluted 10 times and $1 \mathrm{~mL}$ of the diluted bacterial suspension was placed into each well of a Millicell ${ }^{\circledR} 24$ well cell culture plate, containing $2.0 \mathrm{~cm}^{2}$ membrane coupons fixed at the bottom of the wells with the modified side contacting the suspension. The 24 well plate was incubated at $25^{\circ} \mathrm{C}$ for different time intervals $(0.5 \mathrm{~h}, 1 \mathrm{~h}, 2 \mathrm{~h}, 4 \mathrm{~h}$ and $6 \mathrm{~h})$. After incubation, the membrane coupons were gently rinsed twice with $1 \mathrm{~mL}$ of $0.9 \% \mathrm{NaCl}$ solution to remove loosely attached bacteria and sonicated with $2 \mathrm{~mL}$ $0.9 \% \mathrm{NaCl}$ solution for $7 \mathrm{~min}$ to detach the adhered bacteria. The obtained bacterial suspension was then serially diluted with $0.9 \% \mathrm{NaCl}$ and $10 \mu \mathrm{L}$ of each dilution was plated on $\mathrm{LB}$ agar for overnight incubation at $35^{\circ} \mathrm{C}$.

After being exposed to the bacterial suspension, the membrane coupons were also analyzed by FEI Quanta 450 Environmental Scanning Electron Microscope (FE-ESEM) (FEI company, USA) to assess the effects of CuNPs coating on cell morphology of E. coli D21f2. For ESEM observation, membrane samples incubated with bacteria were rinsed, and the bacteria attached on the surface were fixed with $2.5 \%$ glutaraldehyde (in $0.1 \mathrm{mM}$ sodium cacodylate buffer) at ambient temperature for $15 \mathrm{~min}$. Then, the sample was rinsed with $0.1 \mathrm{mM}$ sodium cacodylate buffer solution for twice, and dehydrated with ethanol/DI water solutions: $30 \%, 50 \%, 70 \%, 90 \%, 100 \%$ for 10 min each in trays. Finally, the samples were dried for at least 3 hours and coated with evaporated carbon (Edwards Auto306, UK Crawley) before being analyzed by SEM.

\subsection{Assessment of anti-biofouling performance of the modified membrane}

The anti-biofouling property of the pristine and modified membranes was evaluated using a RO cross-flow filtration system with three types of feed solutions: (i) DI water, (ii) LB solution 
(containing $0.1 \% \mathrm{LB}$ in $10 \mathrm{mM} \mathrm{NaCl}$ ), and (iii) bacterial suspension (containing $10^{5}-10^{6} \mathrm{CFU} / \mathrm{mL}$ in LB solution). The filtration tests with DI water and LB solution were conducted as follows: each membrane was compacted with feed solutions (either with DI water or LB solution) for 8 hours at $27.6 \mathrm{bar}(400 \mathrm{psi})$ to achieve a steady water permeate flux; then, the water permeate flux was continuously monitored for 24 hours with a digital flow meter connected to a personal computer. For the filtration test with bacterial suspension, the LB solution was initially permeated for 8 hours, and then $50 \mathrm{~mL}$ of bacterial suspension $\left(E\right.$. coli $\mathrm{D} 21 \mathrm{f} 2$ in $0.9 \% \mathrm{NaCl}$, at a concentration of $10^{7}-10^{8}$ $\left.\mathrm{CFU} / \mathrm{mL}\left(\mathrm{OD}_{600} \mathrm{~nm}=0.3\right)\right)$ was added and completely mixed with the LB solution to investigate the biofouling propensity of the modified membrane. The normalized flux was obtained by comparing the measured water flux with the initial flux.

\subsection{Regeneration of CuNPs on the membrane surface}

The regeneration potential of the modified membranes was investigated through release-reloading strategy. First, CuNPs needed to be released from the surface of the modified membrane. In order to release the CuNPs, the active side of a freshly modified $10 \mathrm{~cm} \times 10 \mathrm{~cm}$ membrane was put into contact with $32 \mathrm{~g} / \mathrm{L} \mathrm{NaCl}$ solution on a shaker under $50 \mathrm{rpm}$ for 7 days which allowed a near complete release of CuNPs from the membrane surface. The salt solution was replaced each day during the releasing process. After the release of CuNPs, the membrane surface was rinsed with DI water three times to remove the loosely bonded salt ions, then the membrane was soaked in DI water, and stored in a refigrerator until the regeneration process was conducted.

In order to regenerate the (PEI-CuNPs/PAA $)_{10}$ coating, after 7 days of release and subsequent DI water rinse, the membrane was adhered onto a polycarbonate plate, and the same SSLbL coating process was repeated as described in 2.3 . 


\section{Results and Discussion}

\subsection{Characteristics of PEI-CuNPs}

The polyamide active layer of the RO membrane is formed by interfacial polycondensation between trimesoyl chloride (TMC) and m-phenylene diamine (MPD). Due to the hydrolysis of unreacted TMC in aqueous solution, the membrane exhibits a negative charge under general operating $\mathrm{pH}$ conditions. To stably anchor CuNPs onto the membrane surface without adverse effects on its separation performance, PEI was used as a capping agent to provide the particles with a positive charge that then assisted in the binding between the CuNPs and the thin active layer through electrostatic interactions. The amine groups of PEI contain lone pair electrons that will attract $\mathrm{Cu}^{2+}$ in solution and occupy its outer orbit. By adding $\mathrm{NaBH}_{4}$ into the solution, the copper ions are reduced into CuNPs within the matrix of the PEI polymer. The reduced metal nanoparticles exhibited better dispersion ability than CuNPs that were prepared by $\mathrm{CuSO}_{4}$ and $\mathrm{NaBH}_{4}$ without the capping agent (Figure S3).

The average diameter of the synthesized PEI-CuNPs was $39.4 \pm 0.3 \mathrm{~nm}$, as observed by DLS and TEM (Figure S4.A, Figure S4.E). As expected, the PEI-CuNPs remained positively charged in the $\mathrm{pH}$ range of 4 to 10 (Figure S4.B). To confirm that this positive charge was associated with the amine group of PEI, ATR-FTIR spectra was used to investigate the functional units on the CuNP surface (Figure S4.C). Compared with pure CuNPs, the emerging characteristic peaks of an amine group at $1092 \mathrm{~cm}^{-1}$ (C-N stretching), $1594 \mathrm{~cm}^{-1}$ (N-H bending) and a broad band at $\sim 3400 \mathrm{~cm}^{-1}$ (N-H stretching) indicated the successful functionalization of PEI on the PEI-CuNPs' surface. The percentages of CuNPs and polymers in the PEI-CuNPs were evaluated by TGA (Figure S4.D) after being washed (three times) through centrifugation and subsequent drying at $105 \pm 3{ }^{\circ} \mathrm{C}$. The PEICuNPs complex began to decrease in weight with an increase in temperature and remained 
constant after $700{ }^{\circ} \mathrm{C}$. The remaining $57.8 \%$ of the mass consisted of inorganic CuNPs. It is uncertain, though, how much of the copper was oxidized throughout the process, so the mass percentage of CuNPs in the PEI-CuNPs would be within 46.2-57.8\%.

\subsection{Binding PEI-CuNPs on the membrane surface}

CuNPs were incorporated onto the membrane surface through electrostatic interactions with the SSLBL method (Figure 1. B-C). The color of the membrane surface changed to a darker and greener shade as the number of PEI-CuNPs/PAA bilayers increased (Figure S5), and the CuNPs exhibited a uniform distribution on the membrane surface. The freshly synthesized CuNPs were brown, but they were gradually oxidized, turning green, after being exposed to air for approximately five days. The thickness of the LbL films formed on silicon wafers was measured by a profilometer. The thickness was found to increase almost linearly with the increasing number of bilayers, with each PEI-CuNP/PAA bilayer being approximately 60-80 nm (Figure 2.A). In the LbL process, the thickness of the coating can be conveniently controlled through the number of bilayers (Hu and Mi 2013).

XPS analyses of both the pristine and modified membrane surfaces were performed to confirm whether the CuNPs were successfully incorporated. In comparison to a pristine PA membrane, additional signals at $89 \mathrm{eV}$ and $931 \mathrm{eV}$, representing the $\mathrm{Cu} 3 \mathrm{p} 3$ orbital and $\mathrm{Cu} 2 \mathrm{p} / \mathrm{Cu}_{2} \mathrm{O}$, respectively, appeared on the CuNP-modified surface (Figure 2.B). The element contents of $\mathrm{Cu}$ on the modified membrane surface, as assessed by XPS, were $0 \%, 7.3 \pm 1.1 \%, 7.4 \pm 0.7 \%$ and $6.9 \pm 1.5 \%$ (Table S1) for the pristine membrane and the two, six and ten bilayer PEI-CuNP/PAA-modified membranes, respectively. The CuNPs presented very consistent contents on the modified membrane surfaces, and this further suggests that the SSLbL assembly method granted a nearly constant loading of the CuNPs in each bilayer. 
The quantity of CuNPs loaded onto the substrate surface increased with each additional PEICuNP/PAA bilayer. Two bilayers resulted in $1.75 \mu \mathrm{g} \mathrm{cm}^{-2}$ of copper on the membrane surface, and this number increased to $23.7 \mu \mathrm{g} \mathrm{cm}^{-2}$ for a 10 bilayer modified surface (Figure 2.C). By selecting the number of bilayers, the desired quantity of CuNPs on the surface could be achieved. Manual dip-coating was also applied in this study to produce the same PEI-CuNPs/PAA bilayers, while a larger quantity of CuNPs and more uniform bilayers were observed on the membranes modified via SSLbL method in comparison to that manual dipping (Figure S6, S7). The stability of the multi-films has always been a concern in the LbL process. To test the stability of the CuNPs, a modified membrane was placed in a vial that contained $20 \mathrm{~mL}$ DI water, and then submerged in a bath sonicator (Frequencies: $44 \mathrm{kHz} \pm 6 \%$, Branson 8510R-MTH, Branson Ultrasonics Corporation, CT, USA) for 5 min. The quantity of copper on the modified membrane surface remained nearly unchanged before and after sonication (Figure 2.C), indicating a stable binding between the nanoparticles and the membrane surface.

A batch test was performed to further evaluate the amount of released CuNPs and the durability of the 10-bilayer coating. Initially, copper ions were released from the membrane at a rate of 1.25 $\mu \mathrm{g} \mathrm{cm}^{-2}$ day $^{-1}$ under DI wate, and then the release rate declined with operation time (Figure 2.D). After 7 days, the release rate decreased to a level lower than $0.4 \mu \mathrm{g} \mathrm{cm}^{-2}$ day $^{-1}$. The amount of copper that leached out during 7 days of batch testing $\left(7.0 \mu \mathrm{g} \mathrm{cm}^{-2}\right)$ accounted for $29.8 \%$ of the total amount of copper on the membrane. Since the LbL assembly relies heavily on electrostatic interactions between the oppositely charged PEI-CuNPs and PAA layers, a highly concentrated salt solution (i.e., seawater) may disturb the charge balance between the polyelectrolytes, and may cause destabilization of the bilayers resulting in a rapid loss of CuNPs from the membrane. Therefore, the stability of the modfied membrane with (PEI-CuNPs/PAA) ${ }_{10}$ coating was further 
assessed under a higher ionic strength condition $(50 \mathrm{mM} \mathrm{NaCl})$. As observed in Figure 2.D, the release rate of CuNPs showed a slight increase at the mid-ionic strength condition, and near 39.0\% of copper on membrane surface elapsed after seven days. This indicates a comparable stability of the bilayers, even at certain salt concentrations. A slightly increased release rate of $\mathrm{Cu}$ ions may be caused by the increased ionic strength of the solution. The release behavior of the CuNPs under a cross-flow filtration (under 400 psi with DI water for overnight compaction and with $50 \mathrm{mM}$ $\mathrm{NaCl}$ for $24 \mathrm{~h}$ filtration) was also observed (Figure S8), and the varied release rates of CuNPs from the different parts of the membrane was observed. The stability of LbL films under different solution chemistries was also investigated in the existing literatures. Choi et al. presented the electrostatic interactions between the multifilms provided adequate stability to graphene oxide nanosheet modified RO membranes even at high salt concentration $(2000 \mathrm{mg} / \mathrm{L} \mathrm{NaCl})$ and harsh pH (pH 4 and $\mathrm{pH}$ 10) conditions (Choi et al. 2013). In another report it demonstrated that a 10bilayer PAH/PSS (polyallylamine hydrochloride/poly(sodium 4-styrenesulfonate) coating on a polyamide membrane could remain stable in saline water for more than 74 days (Chen et al. 2013).

\section{FIGURE 2.}

\subsection{Characterization of modified membrane}

The ability of a membrane to resist fouling is closely related to its surface physico-chemical properties, particularly the roughness, charge and hydrophobicity. Generally, a membrane with a relatively smooth surface, an electro-neutral and hydrophilic nature presents improved fouling resistance (Rana and Matsuura 2010). AFM, EKA and contact angle measurements were conducted to evaluate the surface physico-chemical property changes of the modified membranes. 
The effect of the CuNP modification on the membrane surface morphology was investigated by SEM, and the corresponding roughness was evaluated using AFM (Figure 3). SEM images presented the characteristic "ridge-and-valley" (Elimelech et al. 1997) structure that resulted from the interfacial polymerization could be clearly identified on a pristine PA membrane surface; however, the valley region was partially filled with nanoparticles and the accompanying polymers after two polyelectrolyte bilayers were added, and a (PEI-CuNPs/PAA) 10 (ten bilayer coating of PEI-CuNPs/PAA) covered the original morphology of the PA membrane completely. This observation can also be noted in the corresponding AFM analysis. The slight increase in roughness (Table S2) of the modified membrane may be associated with the incorporation of CuNPs. Although increasing the surface roughness might cause more bacterial cell deposition on the membrane surface, the deposited bacteria may become inactivated through action of the CuNPs.

\section{FIGURE 3.}

The top layer of the modified membrane mainly consists of PAA, which has the same carboxylic (-COOH) groups as the pristine TFC membrane; therefore, both the pristine and modified membranes exhibited negative charges from $\mathrm{pH} 4.2$ to $\mathrm{pH} 9.9$ (Figure 4.A). It was hypothesized that compared with the pristine membrane, the added PAA layer would significantly increase the number of carboxylic (-COOH) groups on the surface, which may cause a corresponding decrease in the zeta potential for the modified membrane; however, the results show that after (PEI$\mathrm{CuNPs} / \mathrm{PAA})_{10}$ multi-film coating, only a minor decrease in the membrane surface charge was observed. This may be because of the lone pair electrons on the - $\mathrm{NH}_{2}$ group of the PEI beneath the PAA layer that tends to attract some of the protons (isoelectric point of PEI is near pH 10 (Barry R. Bickmore et al. 1999), thereby maintaining nearly the same overall surface charge on the modified membrane. 
CuNP coatings significantly change the membrane surface wettability (Figure 4.B). Because PEI and PAA are naturally hydrophilic, even a ten bilayer polyelectrolyte modified membrane consisting of only PEI and PAA had a contact angle of approximately $20^{\circ}$; while, adding CuNPs to the PEI matrix resulted in a decrease in the surface hydrophilicity (The contact angle increased from $20^{\circ}$ of ten bilayers of PEI/PAA to near $60^{\circ}$ of ten bilayers of PEI-CuNPs/PAA). The CuNPs increased the thickness of the coatings, which might cause additional hydraulic resistance towards permeation of water. However, in the RO desalination process, the applied pressure is typically approximately $13.8-55.2$ bar (200-800 psi), which is adequately high for water molecules to overcome the hydraulic resistance of the coating layers. Thus, the PEI-CuNP/PAA bilayers would not cause an appreciable negative effect on the membrane's performance. This hypothesis was supported by the results of the performance evaluation (Figure 4.C).

Due to higher water flux and excellent salt rejection ability, the TFC membranes are considered to be the 'state-of-the-art' in RO process. Therefore, no significant compromise of membrane performance is deemed after surface modifications. A slight decrease in water permeation flux is observed for the modified membranes with the addition of the coating layers (Figure 4.C and S9). A ten bilayer coating resulted in only a $13.3 \%$ reduction (from $1.54 \pm 0.18 \mathrm{~L} \mathrm{~m}^{-2} \mathrm{~h}^{-1} \mathrm{bar}^{-1}$ of pristine membrane to $1.38 \pm 0.14 \mathrm{~L} \mathrm{~m}^{-2} \mathrm{~h}^{-1} \mathrm{bar}^{-1}$ of the ten-bilayer modified membrane) in the water permeation flux, which might stem from the additional CuNP coatings on the membrane surface. Because no adverse chemical reaction was taking place between polyamide and the coating layer, the modified membrane still performed well, with a high salt rejection capacity.

\section{FIGURE 4.}

\subsection{Antimicrobial and antifouling activities of modified membrane}


To evaluate how the applied surface modification improves the anti-biofouling potential of the membrane, the antimicrobial behavior of different surfaces: pristine, (PEI/PAA)10-, (PEICuNPs/PAA)2-, and (PEI-CuNPs/PAA) ${ }_{10}$-coated membrane, was initially investigated using a model non-pathogenic E. coli. Compared with the polyamide pristine membrane, the membrane coated with CuNPs exhibited strong antibacterial property that increased with the amount of loaded CuNPs on the membrane surface (Figure 5.A). A considerable decrease in CFU of E. coli D21f2 (in the range of $94.3 \%$ to nearly $100 \%$ ) was observed on two, six and ten bilayer PEI-CuNPs/PAA modified membrane surfaces after $1 \mathrm{~h}$ of contact. Although PEI was reported to possess a certain antibacterial property as well (Wong et al. 2012), the pure (PEI/PAA) 10 -modified membrane showed only $14 \%$ inactivation of the bacterial cells attached on the membrane surface. Thus, it could be concluded that CuNPs played the decisive role in the increase of bacterial cell inactivation. FE-ESEM was used to observe the morphology of the bacterial cells on the pristine and (PEI$\mathrm{CuNPs} / \mathrm{PAA})_{2}$ modified surface. The bacaterial inactivation could possibly be evidenced by slight morphological changes (from the regular rod shape) of the deposited bacteria as displayed in Figure 5.B-C, a similar observation was reported in the literature (Ben-Sasson et al. 2014b, Perreault et al. 2014). However, these slight changes in the shape of the bacteria on the SEM images provide only a possible indirect evidence of bacterial inactivation.

\section{FIGURE 5.}

To further evaluate the antimicrobial behavior of the modified membrane, time-dependent static (no pressure, no flow) bacterial inactivation tests were performed using three different strains of bacteria; namely, E. coli D21f2 (Gram negative and non-pathogenic), E. coli O157:H7 (Gram negative and pathogenic) and E. faecalis (Gram positive and pathogenic). The results showed that the (PEI-CuNPs/PAA) ${ }_{10}$-modified membrane exhibited strong anti-bacterial effect to all tested 
bacteria whereas on the pristine membrane, bacteria remained viable with increasing number of CFU over time (Figure 6.A-C). The top layer of the pristine membrane, polyamide, was non-toxic and exhibited relatively rough surface; therefore, the number of bacteria that deposited on the pristine membrane increased significantly with the extension of the contact time between the bacterial suspension and the membrane from 0.5 to 6 hours.

\section{FIGURE 6.}

The strong antimicrobial behavior of the modified membrane is linked to the presence of biocidal CuNPs. The complex mechanism of CuNP action in bacterial cell killing is not fully understood, while some possible cell killing pathways of copper are reported and widely accepted: (1) the appearance of trace amounts of CuNPs depolarizes the cell membrane and causes the anomalous growth of bacteria (Chatterjee et al. 2014); (2) the oxidation of CuNPs results in a release of

electrons and motivates the production of ROS (reactive oxygen species) (Valko et al. 2005), which leads to oxidative damage of the cellular structures; (3) the spontaneous interaction between $\mathrm{Cu}^{2+}$ and phosphorus/sulfur-containing biomolecules (protein, DNA) causes distortion of the organism structure and disruption of metabolism processes (Raffi et al. 2010). It was also reported (Chatterjee et al. 2014) that the interaction between CuNPs and bacterial cells was active, and the binding of the $\mathrm{Cu}^{2+}$ to DNA caused more ion release from CuNPs and led to more DNA damage. This suggests that the interaction between CuNPs and bacterial cells has certain influence on the dissolution rate of the CuNPs. Therefore, one may conclude that a greater number of bilayers should be taken into consideration for the membrane to be effective in bacterial inactivation for longer time periods. 
The anti-biofouling property of the modified membrane was also studied through dynamic RO cross-flow filtration tests using $E$. coli D21f2 as a model bacterium. The permeate flux reductions of the pristine and (PEI-CuNPs/PAA $)_{10}$ coated membranes associated with biofouling are presented in Figure 7.A-B, and the corresponding normalized flux reductions were reported in Figure S10.A. Both the pristine and modified membranes exhibited stable water flux under the DI water condition; but showed a gradual decrease in permeate flux with the LB solution (containing $0.1 \% \mathrm{LB}$ in $10 \mathrm{mM} \mathrm{NaCl}$ ), which is likely caused by deposition of proteins and other constituents of the LB onto the membrane surface. When bacteria were added to the LB solution, the permeate flux of the pristine membrane significantly decreased (66\% reduction after 24 hours of filtration); by contrast, the flux reduction of the CuNP-modified membrane was slower under the same experimental conditions. After 24 hours, a $43 \%$ reduction of permeate flux was observed for the CuNP-modified membrane, which was very close to the control LB solution without bacteria (38\% reduction of the permeate flux). Compared with polyamide surface, this reduced flux decline supported the hypothesis that the CuNPs effectively mitigated the growth of bacterial cells on the membrane surface.

The biofilms formed on both the pristine and the modified membranes after 24 hours filtration in a cross-flow RO cell were visualized via FE-SEM (Figure S10.B-C). Consistent with that observed during the static bacterial inactivation tests, more vegetative cells (with regular rod shape) were visualized on the pristine membrane; while cells with slightly changed morphologies appeared on modified surface. The structures of the biofilm mat produced on the two surfaces also seemed to be different. A thicker matrix and higher concentration of extracellular polymeric substances (EPS) between E.coli cells on the pristine membrane surface may imply that the bacterial communities were embeded within an EPS matrix (Dawson et al. 2012, Shailesh Kumar 2013). In comparison, 
the individual cells on the modified surface were relatively clear, which likely indicates less production of EPS on the CuNPs coated surface. The EPS produced by bacteria on membranes usually reinforces the adhesion between cells and membrane material, and results in the gradual reduction of water flux.

\section{FIGURE 7.}

Both static bacterial adhesion and cross-flow filtration tests demonstrated that CuNPs could significantly improve anti-biofouling property of a polyamide membrane and effectively reduce the permeate flux decline caused by bacterial deposition on the membrane surface. However, it remains unclear whether CuNPs on the membrane surface or dissolved copper ions in solution mainly contributed to this anti-bacterial activity. The interaction between $\mathrm{CuNPs} / \mathrm{Cu}^{2+}$ and bacterial cells and the release rate of CuNPs from the membrane surface requires further study.

\subsection{Regeneration of CuNPs coating on the membrane surface}

Due to the biochemical reactions and the applied shear force during the filtration process, the depletion of biocidal NPs from membrane surface became inevitable for most of the modification method with metallic NPs. Therefore, a convenient and effective method of regeneration of biocidal agents after their discharge should also be taken into consideration. Since the CuNPs were fastened to the membrane through electrostatic interactions, the original structure of the membrane surface was not irreversibly changed. Furthermore, LbL has fewer requirements as relating to substrate surface properties, and it could be successfully produced on different substrates such as silicon, glass or quartz plates (Hyder et al. 2011) (Nogueira et al. 2011). Thus, it is expected that the PEI-CuNPs/PAA multi-film could easily be regenerated by the same SSLbL technique once CuNPs are completely depleted from the modified membrane. 
To confirm the regeneration of PEI-CuNPs/PAA bilayers after their depletion, a freshly modified membrane was immersed in $32 \mathrm{~g} / \mathrm{L} \mathrm{NaCl}$ solution for seven days to allow a near-complete release of CuNPs, and then multi-film was regenerated via the same SSLbL method. It was observed that the quantity of CuNPs after (PEI-CuNPs/PAA) 10 regeneration was even higher than the freshly modified membrane, which may be because of the incomplete release of CuNPs after seven days. Owing to the high quantity of CuNPs content, the regenerated membrane exhibited excellent antimicrobial properties, and almost complete inactivation (nearly $100 \%$ decrease in CFU when compared with that of pristine membrane) was observed with the regenerated membranes. This observation supports that the potential anti-biofouling properties of the modified membrane could be maintained during long-term operation through a process of regeneration of the CuNPs coating after being depleted.

\section{FIGURE 8.}

There is no denying that the CuNPs are more prone to oxidation and release when compared with AgNPs; while the LbL process benefits from the increased numbers of CuNPs on the membrane surface, and the (PEI-CuNPs/PAA) 10 modified membrane showed a comparable antimicrobial performance and NP durability in comparison to that of AgNPs modified surfaces, according to our previous work (Soroush et al. 2015, Rahaman et al. 2014). The released CuNP could also potentially serve as bacteria-inactivating NPs/ions in the feed solution, inhibiting the reproduce of bacteria.

Compared with manual LbL process reported in several references, the SSLbL process introduced in this study could produce a more uniform layer on the membrane surface within a very short period. Even though the spinning process is difficult to apply in large scale membrane modification, 
it could be easily replaced by a rolling spiral axis, or the static spray nozzles could be changed into moving ones to produce a uniform layer for industrial applications. Therefore, the scaling up of this technique for large-scale industrial production would not be an issue. Furthermore, the LbL process is a facile method for surface modification, and it does not have critical requirements for the substrates. Thus, even though NPs deplete after long-time use, the functional units could be conveniently reproduced on the membrane surface via the same method. In the case of membranes in a spiral wound module, the combining the SSLbL coating of CuNPs at the beginning, and reduction of $\mathrm{Cu}$ ions or the dipping LbL process for the regeneration of CuNPs in situ could be a feasible way to improve the anti-biofouling performance of the RO membrane with the lower cost when compared with the one with the AgNPs as the modifier.

\section{Conclusions}

To improve the antimicrobial properties of RO membrane surfaces, CuNPs were used as biocides and deposited on a membrane via 'spray- and spin-assisted layer-by-layer' self-assembly. This rapid (32 sec per bilayer) and efficient method improved the uniformity of the CuNPs distribution on a modified membrane's active layer compared to conventional dip coating techniques.

In general, the LbL modification did not adversely affect the membrane's separation performance and maintained the same level of salt rejection after modification. However, additional bilayers reduced the surface's hydrophilicity and resulted in a $13.3 \%$ reduction in water permeation flux.

The CuNP-functionalized membrane exhibited a significant inactivation ability to model Gram positive and Gram negative bacteria. The inactivation of bacteria increased with increasing CuNP loading on the membrane surface. The CuNP-modified membrane exhibited ability to inhibit 
bacterial growth on the membrane surface and thus reduce the permeate flux decline (caused by biofouling) considerably.

The quantity of CuNPs and the bacterial inactivation properties of the membrane could be maintained through regeneration of PEI-CuNPs/PAA multi-films via the same SSLbL method after the depletion of CuNPs.

Overall, both the effective and time-saving modification process along with the promising antimicrobial results demonstrate the potential for this novel surface coating in practical applications for membrane biofouling control. 


\section{Acknowledgments}

The authors acknowledge the Natural Sciences and Engineering Research Council (NSERC) of Canada for providing funding support for this project. Wen Ma acknowledges the support of a Helene Linder Doctoral Fellowship from Concordia University. N.T. acknowledges the support of the CRC program. 


\section{References}

Rahaman, M.S., Vecitis, C.D. and Elimelech, M. (2012) Electrochemical carbon-nanotube filter performance toward virus removal and inactivation in the presence of natural organic matter. Environmental Science \& Technology 46(3), 1556-1564.

Greenlee, L.F., Lawler, D.F., Freeman, B.D., Marrot, B. and Moulin, P. (2009) Reverse osmosis desalination: water sources, technology, and today's challenges. Water Research 43(9), 2317-2348.

Elimelech, M. and Phillip, W.A. (2011) The future of seawater desalination: energy, technology, and the environment. Science 333(6043), 712-717.

Kang, G.D. and Cao, Y.M. (2012) Development of antifouling reverse osmosis membranes for water treatment: A review. Water Res 46(3), 584-600.

Rana, D. and Matsuura, T. (2011) Surface modification for antifouling membrane. Chemical Reviews 110, 2448-2471.

Mi, B. and Elimelech, M. (2010) Organic fouling of forward osmosis membranes: Fouling reversibility and cleaning without chemical reagents. Journal of Membrane Science 348(1-2), 337-345.

Coneski, P.N., Weise, N.K., Fulmer, P.A. and Wynne, J.H. (2013) Development and evaluation of selfpolishing urethane coatings with tethered quaternary ammonium biocides. Progress in Organic Coatings 76(10), 1376-1386.

Ma, W., Rahaman, M.S. and Therien-Aubin, H. (2015) Controlling biofouling of reverse osmosis membranes through surface modification via grafting patterned polymer brushes. Journal of Water Reuse and Desalination 5(3), 326-334.

Ben-Sasson, M., Zodrow, K.R., Genggeng, Q., Kang, Y., Giannelis, E.P. and Elimelech, M. (2014a) Surface functionalization of thin-film composite membranes with copper nanoparticles for antimicrobial surface properties. Environmental Science \& Technology 48(1), 384-393.

Tiraferri, A., Vecitis, C.D. and Elimelech, M. (2011) Covalent binding of single-walled carbon nanotubes to polyamide membranes for antimicrobial surface properties. ACS Applied Materials \& Interfaces 3(8), 28692877.

Vecitis, C.D., Schnoor, M.H., Rahaman, M.S., Schiffman, J.D. and Elimelech, M. (2011) Electrochemical multiwalled carbon nanotube filter for viral and bacterial removal and inactivation. Environmental Science \& Technology 45(8), 3672-3679.

He, L., Dumée, L.F., Feng, C., Velleman, L., Reis, R., She, F., Gao, W. and Kong, L. (2015) Promoted water transport across graphene oxide-poly(amide) thin film composite membranes and their antibacterial activity. Desalination 365, 126-135. 
Das, M.R., Sarma, R.K., Saikia, R., Kale, V.S., Shelke, M.V. and Sengupta, P. (2011) Synthesis of silver nanoparticles in an aqueous suspension of graphene oxide sheets and its antimicrobial activity. Colloids and Surfaces B: Biointerfaces 83(1), 16-22.

Mansouri, J., Harrisson, S. and Chen, V. (2010) Strategies for controlling biofouling in membrane filtration systems: challenges and opportunities. Journal of Materials Chemistry 20(22), 4567-4586.

Soroush, A., Ma, W., Silvino, Y. and Rahaman, M.S. (2015) Surface modification of thin film composite forward osmosis membrane by silver-decorated graphene-oxide nanosheets. Environ. Sci.: Nano 2, 395 - 405.

Soroush, A., Ma, W., Cyr, M., Rahaman, M.S., Asadishad, B. and Tufenkji, N. (2016) In SituSilver Decoration on Graphene Oxide-Treated Thin Film Composite Forward Osmosis Membranes: Biocidal Properties and Regeneration Potential. Environmental Science \& Technology Letters 3(1), 13-18.

Ben-Sasson, M., Lu, X., Nejati, S., Jaramillo, H. and Elimelech, M. (2016) In situ surface functionalization of reverse osmosis membranes with biocidal copper nanoparticles. Desalination 388, 1-8.

Ahamed, M., Alhadlaq, H.A., Khan, M.A.M., Karuppiah, P. and Al-Dhabi, N.A. (2014) Synthesis, characterization, and antimicrobial activity of copper oxide nanoparticles. J. Nanomater. 2014, 1-4.

Dwidjosiswojo, Z., Richard, J., Moritz, M., Dopp, E., Flemming, H.-C. and Wingender, J. (2011) Influence of copper ions on the viability and cytotoxicity of Pseudomonas aeruginosa under conditions relevant to drinking water environments. International Journal of Hygiene and Environmental Health 214(6), 485-492.

Morton, S.W., Herlihy, K.P., Shopsowitz, K.E., Deng, Z.J., Chu, K.S., Bowerman, C.J., Desimone, J.M. and Hammond, P.T. (2013) Scalable manufacture of built-to-order nanomedicine: spray-assisted layer-by-layer functionalization of PRINT nanoparticles. Advanced Materials 25(34), 4707-4713.

Liu, Y., Rosenfield, E., Hu, M. and Mi, B. (2013) Direct observation of bacterial deposition on and detachment from nanocomposite membranes embedded with silver nanoparticles. Water Research 47(9), 2949-2958.

Dumée, L.F., He, L., King, P.C., Moing, M.L., Güller, I., Duke, M., Hodgson, P.D., Gray, S., Poole, A.J. and Kong, L. (2015) Towards integrated anti-microbial capabilities: Novel bio-fouling resistant membranes by high velocity embedment of silver particles. Journal of Membrane Science 475, 552-561.

Mauter, M.S., Wang, Y., Okemgbo, K.C., Osuji, C.O., Giannelis, E.P. and Elimelech, M. (2011) Antifouling ultrafiltration membranes via post-fabrication grafting of biocidal nanomaterials. ACS Appl Mater Interfaces 3(8), 2861-2868.

Rajesh, S., Yan, Y., Chang, H.-C., Gao, H. and Phillip, W.A. (2014) Mixed mosaic membranes prepared by layer-by-layer assembly for ionic separations. ACS Nano 8(12), 12338-12345.

Chen, L., Thérien-Aubin, H., Wong, M.C.Y., Hoek, E.M.V. and Ober, C.K. (2013) Improved antifouling properties of polymer membranes using a 'layer-by-layer' mediated method. Journal of Materials Chemistry B $1(41), 5651-5658$. 
Gittleson, F.S., Hwang, D., Won-Hee Ryu, Hashmi, S.M., Hwang, J., Goh, T. and Taylor, A.D. (2015) Ultrathin nanotube/nanowire electrodes by spin spin-spray layer-by-layer assembly: a concept for transparent energy storage 2015. ACS Nano 9(10), 10005-10017.

Gittleson, F.S., Kohn, D.J., Li, X. and Taylor, A.D. (2012) Improving the assembly speed,quality, and tunability of thin conductive multilayers. ACS Nano 6(5), 3703-3711.

$\mathrm{Hu}, \mathrm{M}$. and Mi, B. (2013) Enabling graphene oxide nanosheets as water separation membranes. Environmental Science \& Technology 47(8), 3715-3723.

Choi, W., Choi, J., Bang, J. and Lee, J.H. (2013) Layer-by-layer assembly of graphene oxide nanosheets on polyamide membranes for durable reverse-osmosis applications. ACS Applied Materials \& Interfaces 5(23), $12510-12519$.

Rana, D. and Matsuura, T. (2010) Surface Modifications for Antifouling Membranes. Chemical Reviews 110, 2448-2471.

Elimelech, M., Zhu, X., Childress, A.E. and Hong, S. (1997) Role of membrane surface morphology in colloidal fouling of cellulose acetate and composite aromatic polyamide reverse osmosis membranes. Journal of Membrane Science 127, 101-109.

Barry R. Bickmore, Michael F. Hochella, J., Dirk Bosbach and Charlet, L. (1999) Methods for performing atomic force microscopy imaging of clay minerals in aqueous solutions. Clays and Clay Minerals 47(5), 573581.

Wong, S.Y., Han, L., Timachova, K., Veselinovic, J., Hyder, M.N., Ortiz, C., Klibanov, A.M. and Hammond, P.T. (2012) Drastically lowered protein adsorption on microbicidal hydrophobic/hydrophilic polyelectrolyte multilayers. Biomacromolecules 13(3), 719-726.

Ben-Sasson, M., Lu, X., Bar-Zeev, E., Zodrow, K.R., Nejati, S., Qi, G., Giannelis, E.P. and Elimelech, M. (2014b) In situ formation of silver nanoparticles on thin-film composite reverse osmosis membranes for biofouling mitigation. Water Research 62, 260-270.

Perreault, F., Tousley, M.E. and Elimelech, M. (2014) Thin-Film Composite Polyamide Membranes Functionalized with Biocidal Graphene Oxide Nanosheets. Environmental Science \& Technology Letters 1(1), 71-76.

Chatterjee, A.K., Chakraborty, R. and Basu, T. (2014) Mechanism of antibacterial activity of copper nanoparticles. Nanotechnology 25(13), 1-12.

Valko, M., Morris, H. and Cronin, M.T.D. (2005) Metals, toxicity and oxidative stress. Current Medicinal Chemistry 12, 1161-1208.

Raffi, M., Mehrwan, S., Bhatti, T.M., Akhter, J.I., Hameed, A., Yawar, W. and ul Hasan, M.M. (2010) Investigations into the antibacterial behavior of copper nanoparticles against Escherichia coli. Annals of Microbiology 60(1), 75-80. 
Dawson, L.F., Valiente, E., Faulds-Pain, A., Donahue, E.H. and Wren, B.W. (2012) Characterisation of clostridium difficile biofilm formation, a role for Spo0A. PloS One 7(12), e50527.

Shailesh Kumar, M.C. (2013) Role of CSE1034 in Escherichia coli Biofilm Destruction. Journal of Microbial \& Biochemical Technology 05(03).

Hyder, M.N., Hammond, P.T., Lee, S.W., Cebeci, F.C., Schmidt, D.J., Shao-Horn, Y. and Hammond, P.T. (2011) Layer-by-layer assembled polyaniline nanofiber/multiwall carbon nanotube thin film electrodes for highpower and high-energy storage applications. ACS Nano 5(11), 8552-8561.

Nogueira, G.M., Banerjee, D., Cohen, R.E. and Rubner, M.F. (2011) Spray-layer-by-layer assembly can more rapidly produce optical-quality multistack heterostructures. Langmuir 27(12), 7860-7867.

Rahaman, M.S., Thérien-Aubin, H., Ben-Sasson, M., Ober, C.K., Nielsen, M. and Elimelech, M. (2014) Control of biofouling on reverse osmosis polyamide membranes modified with biocidal nanoparticles and antifouling polymer brushes. Journal of Materials Chemistry B 2(12), 1724-1732. 